\title{
Estimating quality factor and mean grain size of sediments from high-resolution marine seismic data
}

\author{
Luke J. W. Pinson ${ }^{1}$, Timothy J. Henstock ${ }^{1}$, Justin K. Dix ${ }^{1}$, and Jonathan M. Bull ${ }^{1}$
}

\begin{abstract}
Seismic quality factor has the potential to characterize sediment properties but seldom is used by the industry for offshore site investigations because of practical difficulties with reflection seismology (e.g., restricted bandwidth) and because of uncertainties in rock-physics models. A spectral-ratio analysis of highresolution marine seismic data can determine a quality factor to within a $95 \%$ confidence of \pm 10 within the uppermost $30 \mathrm{~m}$ of unconsolidated marine sediments. Our spectral-ratio technique does not require assumptions on how attenuation scales with frequency. Emphasis is placed on interpretation of spectral signatures before applying an iteratively reweighted robust leastsquares regression to subdue the effects of noise and local heterogeneities when determining the quality factor of a sediment package. We combined data from boomer and chirp sources to
\end{abstract}

examine attenuation over four octaves of frequency (0.5-8.0 $\mathrm{kHz}$ ) and to demonstrate that expanding the frequency range improves the precision and accuracy of quality-factor fits. We obtain frequency-independent quality factors with $95 \%$ confidence intervals of $135(+12 ;-10)$ and $107(+6 ;-5)$ for silty clays with mean grain sizes of 7.7 and 6.9 phi, respectively, and 63 $(+10 ;-7)$ for a modern sand deposit with mean grain size 2.5 phi, from the Solent (U. K.). Sediments with higher quality factors require more independent observations to achieve a desirable $95 \%$ confidence. We required only 45 traces over sands and 1250 traces over the lowest attenuating silty clays. By constructing an empirical model of quality factor against mean grain size from published sediment studies, the mean grain sizes of our Solent sediments can be located, and we find that quality factor can be used to distinguish between coarse grain-dominated and claydominated sediments.

\section{INTRODUCTION}

Remote measurements of compressional-wave attenuation, in contrast to intrusive methods (e.g., coring and placing instruments in situ), can be obtained over large areas quickly and cheaply and are repeatable because they are nondestructive to sediments and their in situ properties. If in situ compressional-wave attenuation can be related to physical properties such as grain size, this would be cost-effective and would provide valuable information for offshore construction and aggregate industries. This paper details how to derive a quality factor $(Q)$ with a desired statistical precision for shallow sediment packages by using high-resolution seismic-reflection data, and shows how such estimates can be related to mean grain sizes of unconsolidated sediments.

Seismic waves attenuate as they propagate in ways that are characteristic of the media, with energy being lost through intrinsic atten- uation and scattering. There are two main theories for intrinsic attenuation in saturated, unconsolidated marine sediments. Biot-Stoll (Biot, 1956a, 1956b; Stoll, 1974) and local flow theories (Dvorkin and Nur, 1993) assume that primary energy losses are through viscous dissipation and predict a nonlinear relationship between compressional-wave attenuation and frequency. The grain-sliding (G-S) theory (Buckingham, 2000) assumes that grain-to-grain shearing produces microscopic frictional losses and predicts a near-linear scaling of compressional-wave attenuation with frequency.

The Biot-Stoll model shows a marked velocity dispersion and nonlinear transition in compressional-wave attenuation from low to high asymptotic values between $50 \mathrm{~Hz}$ and $10 \mathrm{kHz}$, particularly in coarser granular sediment (Stoll, 2002). Observations from the Sediment Acoustics Experiment of 1999 (SAX99) (Thorsos et al., 2001) and the crosshole tomography experiments of Turgut and Yamamoto

Manuscript received by the Editor 2 July 2007; revised manuscript received 26 January 2008; published online 24 June 2008.

${ }^{1}$ University of Southampton, National Oceanography Centre, Southampton, U. K. E-mail: 1.pinson@soton.ac.uk; then@noc.soton.ac.uk; jkd@noc.soton. ac.uk;jmb1@noc.soton.ac.uk.

(C) 2008 Society of Exploration Geophysicists. All rights reserved. 
(1990) are generally in agreement with the Biot-Stoll model (see Buchanan, 2006).

However, the near-linear scaling of compressional-wave attenuation with frequency and logarithmic velocity dispersion of the G-S model conforms better with the SAX99 data above $10 \mathrm{kHz}$. The G-S model also relates better to other observations in a range of sediments within low-frequency seismic surveys and high-frequency laboratory studies (e.g., Hamilton, 1972; Bowles, 1997; Simpson and Houston, 2000; Williams et al., 2002; Simpson et al., 2003). The G-S theory suggests that intrinsic attenuation is independent of viscous flow parameters such as permeability, tortuosity, and the structure factor and is governed instead by grain size, porosity, and overburden pressure.

Scattering produces frequency-dependent attenuation (O'Doherty and Anstey, 1971; Hosken et al., 1992; Muller and Shapiro, 2004). Multiple backscattering is a function of the vertical correlation between scatterers relative to the seismic wavelength (Shapiro and Hubral, 1999). Random diffraction and refraction effects are functions of the finite horizontal extent of inhomogeneities relative to the Fresnel zone of the incident wave (Muller and Shapiro, 2004). Scattering sedimentary layers can be identified within high-resolution seismic data and can be rejected for further quantitative examination (e.g., Schock, 2004a). We use this approach because our interest lies in determining the sediment physical properties that relate to intrinsic attenuation rather than the sediment package structures that cause scattering attenuation.

Previous studies have used high-resolution chirp sources to estimate compressional-wave attenuation in near-surface sediments remotely (LeBlanc et al., 1992; Stevenson et al., 2002; Schock, 2004a, 2004b). LeBlanc et al. (1992) developed an attenuation-based classification model that relates the relaxation time of sediments to the shift in instantaneous frequency of the seismic signal. Stevenson et al. (2002) developed this method by estimating the instantaneous frequency shift only from maximum amplitudes (i.e., from interpolating between seabed and subbottom interface reflections). Their analyses assume a linear scaling of compressional-wave attenuation with frequency and require a specific chirped pulse that will remain zero phase before and after dispersion and attenuation (LeBlanc et al., 1992). Williams et al. (2002) and Schock (2004a) use a spectralratio method on maximum amplitudes to approximate the compressional-wave attenuation for an input of the first iteration of an inversion based on the Biot model (Biot, 1956a, 1956b). The scaling of compressional-wave attenuation is near linear with frequency between $4 \mathrm{kHz}$ and $8 \mathrm{kHz}$ (Schock, 2004a). The data of Williams et al. (2002), who use a range of additional methods from SAX99, arguably show the same relationship between $2 \mathrm{kHz}$ and $400 \mathrm{kHz}$. Schock (2004a) speculates that an increasing scattering attenuation counters the decreasing intrinsic attenuation predicted by the Biot model, whereas Williams et al. (2002) additionally suggest that a combination of Biot and G-S theory is possible.

We accept that the relationship between frequency and compressional-wave attenuation is still ambiguous, and we will not constrain ourselves with assumptions required for a full sediment-model inversion. Instead, we evaluate how attenuation varies with frequency within unconsolidated marine sediments using our high-resolution (broadband) seismic-reflection sonars $(0.5-8.0 \mathrm{kHz})$. We use an adaptation of the spectral-ratio method (Båth, 1974) and do not require assumptions on how compressional-wave attenuation varies with frequency. We identify trends in attenuation with frequency within spectral ratios and assign a quality factor $\left(Q_{S R}\right)$ to them by fitting a curve using iteratively reweighted robust least-squares regression.

In this study, we demonstrate repeatable significant $Q_{S R}$ fits with $95 \%$ confidence intervals of approximately \pm 10 within known unconsolidated sediments from the Solent (U. K.). We also combine data from separate boomer and chirp high-resolution sources over the same location to observe $Q_{S R}$ over more than an order of magnitude variation in frequency, or more than four octaves. We have chosen three unconsolidated sediment packages to demonstrate that fewer observations are required to achieve our desired level of confidence in $Q_{S R}$ for higher-attenuating coarse grain-dominated sediments than for lower-attenuating clays. We assess our results by relating the $Q_{S R}$ of our Solent sediments to their known mean grain sizes using an existing empirical model.

\section{SPECTRAL-RATIO CALCULATION}

The spectral-ratio method (Båth, 1974; Jannsen et al., 1985) has been adapted for application to seismic-reflection data so that compressional-wave attenuation is measured explicitly as a function of two-way time, as opposed to distance and velocity. Within the seismic-reflection data, we require a subbottom subhorizontal reflection that forms the lower boundary of a laterally consistent sediment package beneath the seabed. We assume that within our experimental frequency range $(0.5-8.0 \mathrm{kHz})$, spherical divergence and reflection coefficients are independent of frequency.

Allowing the compressional-wave attenuation coefficient $\alpha(f)$ and the quality factor $Q(f)$ to be functions of frequency $f$, their relationship is defined as

$$
\alpha(f)=\frac{\pi \cdot f}{c(f) \cdot Q(f)}=\frac{\pi \cdot f \cdot \Delta t(f)}{X \cdot Q(f)},
$$

where $c(f)$ is the phase velocity, $\Delta t(f)$ the two-way time, and $X$ the distance traveled within the attenuating medium. By attenuating the unknown amplitude $A_{0}(f)$, emitted from a seismic source at distance and time zero, the amplitude of the seabed reflection $A_{S}(f)$ for some frequency, at sonar-seabed depth $X_{S}$, and seawater attenuation $\alpha_{S}(f)$, is given as

$$
\left|A_{S}(f)\right|=\left|A_{0}(f) \cdot G_{S} \cdot R_{S}\right| \cdot \exp \left(-2 \cdot \alpha_{S}(f) \cdot X_{S}\right),
$$

assuming that $R_{S}$, the unknown seabed reflection coefficient, and $G_{S}$, the unknown spherical divergence within the water column, are independent of frequency.

Similarly, where $R_{R}$, the unknown reflection coefficient from a subbottom interface, and $G_{R}$, the unknown spherical divergence beneath the seabed, are independent of frequency, the amplitude of the reflection from the subbottom interface $A_{R}(f)$, for some frequency at subbottom depth $X_{R}$, and sediment attenuation $\alpha_{R}(f)$, is given as

$$
\begin{aligned}
\left|A_{R}(f)\right|= & \left|A_{0}(f) \cdot G_{S} \cdot G_{R} \cdot\left(1-R_{S}^{2}\right) \cdot R_{R}\right| \\
& \cdot \exp \left(-2 \cdot \alpha_{S}(f) \cdot X_{S}\right) \cdot \exp \left(-2 \cdot \alpha_{R}(f) \cdot X_{R}\right) .
\end{aligned}
$$

By assuming normal incidence, the two-way transmission coefficient across the seabed interface in equation 3 is represented as (1 $-R_{S}^{2}$ ). The unknown components $A_{0}(f), G_{S}$ and all the unknown responses from the source and receiver that have not been shown can be eliminated by taking the ratio of $A_{R}(f)$ and $A_{S}(f)$ : 


$$
\left|\frac{A_{R}(f)}{A_{S}(f)}\right|=\left|\frac{G_{R} \cdot\left(1-R_{S}^{2}\right) \cdot R_{R}}{R_{S}}\right| \cdot \exp \left(-2 \cdot \alpha_{R}(f) \cdot X_{R}\right) .
$$

By substituting for $\alpha_{R}(f)$ in equation 4 using equation 1 where $\Delta t_{R}(f)$ is the two-way time within the sediment, we obtain

$$
\left|\frac{A_{R}(f)}{A_{S}(f)}\right|=\left|\frac{G_{R} \cdot\left(1-R_{S}^{2}\right) \cdot R_{R}}{R_{S}}\right| \cdot \exp -\frac{\pi \cdot f \cdot \Delta t_{R}(f)}{Q(f)} .
$$

Taking the natural log of equation 5 gives

$$
\ln \left|\frac{A_{R}(f)}{A_{S}(f)}\right|=\ln \left|\frac{G_{R} \cdot\left(1-R_{S}^{2}\right) \cdot R_{R}}{R_{S}}\right|-\frac{\pi \cdot f \cdot \Delta t_{R}(f)}{Q(f)},
$$

where all unknown components have become separated from measurable components and $Q(f)$. Unknown components $G_{R}, R_{S}$, and $R_{R}$ can be assumed to be constant from trace to trace if interfaces are subhorizontal, seismic energy is normally incident on them, and sediment properties are laterally consistent.

These conditions minimize energy losses through transmission losses (refraction and critical angles) and mode conversion, and preserve amplitudes generated by the two interfaces and the amount of spherical divergence that occurs between them. If we measure amplitudes reflected from isolated single-planar interfaces, possible focusing effects from irregular interfaces and tuning effects with reflections from neighboring interfaces are removed, and we can assume that the unknown components are independent of frequency.

However, by causality, velocity dispersion occurs if attenuation is present in the propagation, which could cause reflection coefficients to vary with frequency. According to the Kramers-Kronig relation, for a constant $Q$ velocity dispersion is only logarithmic, and as long as $Q$ remains greater than 20, there will be a change of less than $5 \%$ in the seabed reflection coefficient, which we consider negligible. The lowest $Q$ commonly encountered is that of coarse sands, which tends to be about 30 (Hamilton, 1972; Guigné et al., 1989; Stevenson et al., 2002). Considering a frequency-dependent $Q_{S R}$, the Biot model predicts a variation in $R_{S}$ with frequency of no more than $5 \%$ within our experimental frequency range (Schock, 2004a), which we also consider negligible. Thus, by plotting the natural $\log$ of $\left|A_{R}(f) / A_{S}(f)\right|$ as a function of $\pi \cdot f \cdot \Delta t_{R}(f)$ and fitting an appropriate curve using leastsquares regression, the relationship of $1 / Q(f)$ with frequency can be assessed.

It is inconsequential whether $1 / Q(f)$ is independent or dependent on frequency as it is found by simply differentiating the curve. Equally, a straight-line fit implies that a frequency-independent $Q_{S R}$ is appropriate for the data set. The precision in curve fitting can be improved by plotting the results from many traces taken over the same interfaces. Any linear variation in attenuation from small changes in sediment thickness between the subhorizontal interfaces is accounted for by considering $\Delta t_{R}(f)$. Small changes in $\Delta t_{R}(f)$ also will incur equivalent changes in $G_{R}$, which combines with other unknown frequency-independent constants to form the intercept of the curve. Variations in $G_{R}$ are frequency independent. They produce only small changes in the value of the intercept that average out during regression and do not affect gradients of the fitted curve.

\section{HIGH-RESOLUTION SEISMIC SOURCES}

The logarithmic dependence of attenuation with frequency requires a large frequency range to assess the relationship between the two. Hence, we use high-resolution seismic-reflection data, and where possible, we combine data from boomer and chirp sources over the same location to produce an extended frequency range. Boomer sources consist of an insulated metal plate that is repelled explosively by a magnetic field. This motion generates a broadband pulse that peaks near $1 \mathrm{kHz}$ and is repeatable (e.g., Simpkin and Davis, 1993). The effective-power spectrum above $-30 \mathrm{~dB}$ spans approximately $0.25-4.0 \mathrm{kHz}$ (Figure 1a). Chirp profilers produce a digital, highly repeatable, broadband pulse, making such profilers ideal for qualitative and quantitative examination of the subseabed down to approximately 30 m (e.g., Schock and LeBlanc, 1990; Bull et al., 1998; Stevenson et al., 2002).

In this study, we calibrate our chirp sonar to produce a linear sweep with a sine-squared taper function covering one-eighth of the time duration (Gutowski et al., 2002). The wide effective-power spectrum exhibited by the chirp source spans 2-11 kHz (Figure 1b) and facilitates improved vertical and spectral resolution over the boomer source. Therefore, by combining boomer and chirp data, attenuation can be measured over four octaves of frequency (e.g., $0.5-8.0 \mathrm{kHz}$ ) or more. Streamers are towed directly behind the sources. The streamer used with the boomer source comprises seven hydrophone elements spaced across $1 \mathrm{~m}$, and the streamer used with the chirp source comprises eight hydrophone elements spaced across $1 \mathrm{~m}$.

\section{SPECTRAL-RATIO TECHNIQUE}

\section{Selection of sediment packages}

We must adhere to the criteria established for equation 6 when selecting suitable sediment packages to analyze. We require a consistent subhorizontal subbottom reflector and a consistent subhorizontal seabed reflection, both without any neighboring reflections within 1 to $2 \mathrm{~ms}$, to avoid tuning effects. The target reflection must be shallower than the first seabed multiple, reducing the possibility of delayed energy affecting the spectra. Using multiple traces that sample the reflectors can improve curve fitting by averaging out noise and the effects of local inhomogeneities, if there is no lateral variation in the sediments. Including lateral variations will decrease the a)

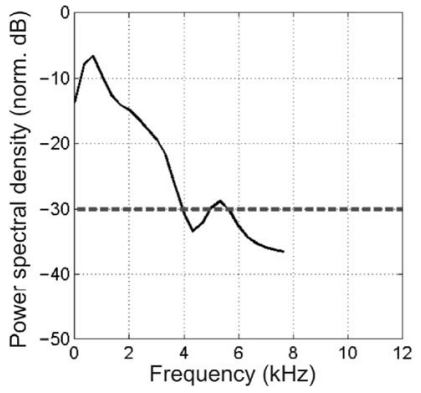

b)

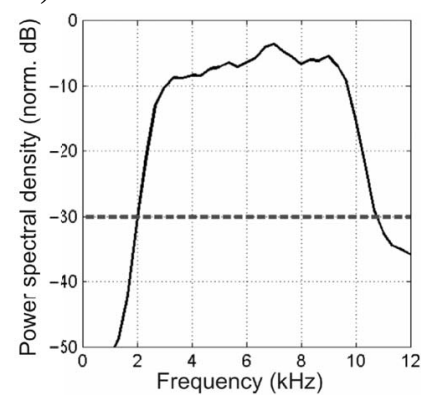

Figure 1. Source power spectra recovered from the seabed reflection at location A in this study: (a) boomer and (b) chirp. The power spectra are normalized and then smoothed over a $100-\mathrm{Hz}$ window. 
precision in the $Q_{S R}$ assigned to a sediment package because attenuation, spherical divergence, and reflection coefficients might vary from trace to trace.

In this study, we examine three sediment packages from the Solent, shown in Figure 2. The seabed and subbottom lithology is determined from a combination of core samples and investigations by West (1980) and by Velegrakis (2000). The mean grain size is reported as phi, which is a logarithmic scale representing grain diameter

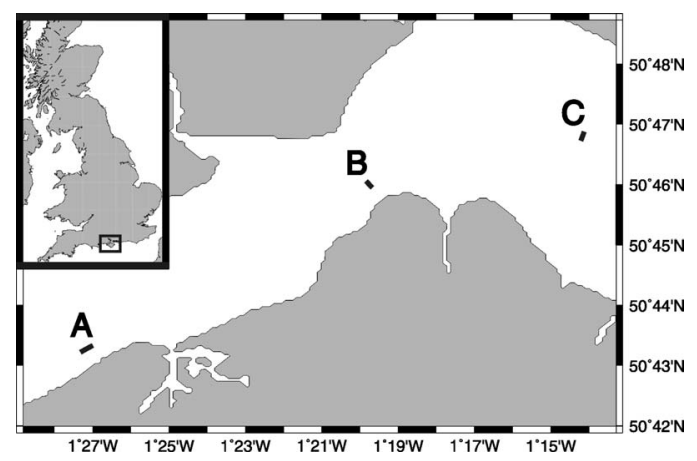

Figure 2. Map locating survey lines over sediment packages at locations A, B, and C in the Solent, U. K. $(\mathrm{mm})\left(\mathrm{phi}=-\log _{2}(\mathrm{~mm})\right)$. The sorting is represented as the range from the mean phi to the 16th and 84th percentile of the grain-size distribution.

The sediment package at location A (Figure $3 \mathrm{a}$ and $\mathrm{b}$ ) consists of a thin layer of gravel dunes approximately 1-2 m thick, overlying the Bembridge Marls formation (silty clays), which have a mean grain size and sorting of 7.7 phi $(+1.3 ;-3.1)$, determined from standard nomenclature analysis of a 17-m core (J. Dean, personal communication, 1995). Three surveys were carried out over the sediment package at location A: a southwest-northeast chirp survey in tidalflow direction (750 traces); a northeast-southwest chirp survey against tidal-flow direction (1250 traces); and a single boomer survey (125 traces).

The sediment package at location B (Figure 3c) consists of a veneer of sand overlying some of the Headon Beds, which include shelly clays with some sands. Based on data from an adjacent borehole, this portion of the Headon Beds has a mean grain size and sorting of 6.9 phi $(+2.1 ;-1.5)$. The sediment package at location C (Figure 3d) is known as Brambles Bank and consists of unconsolidated sands with a mean grain size and sorting of 2.5 phi $(+0.3 ;-0.3)$, as determined by a seabed grab. The Brambles Bank sands overlay the more consolidated Barton Sand beds, which form the subbottom interface. Single chirp surveys were carried out over sediment packages at locations $\mathrm{B}$ and $\mathrm{C}$, and the analyses use 400 and 45 traces, respectively. a)

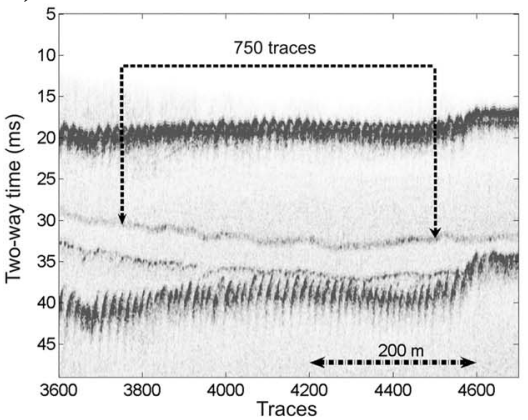

b)

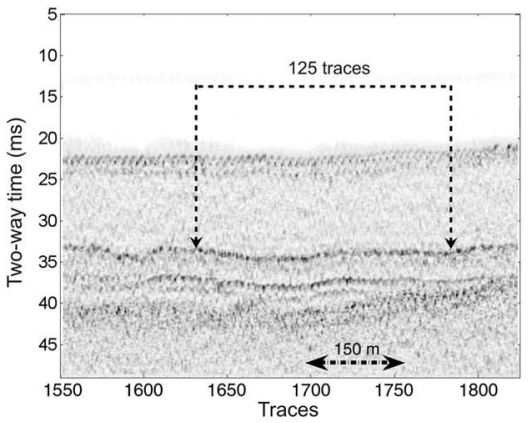

c)

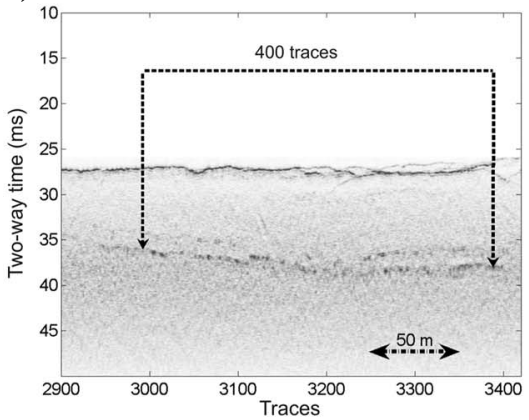

d)

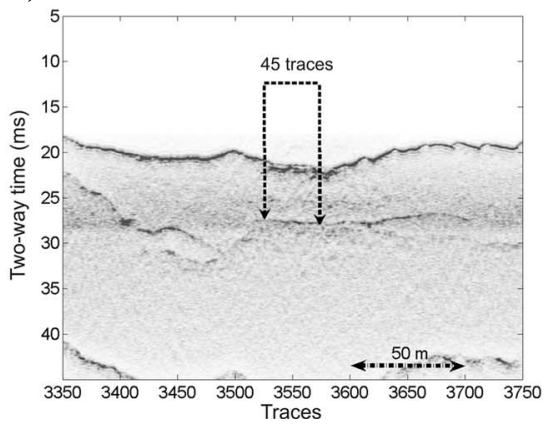

Figure 3. (a) Chirp profile over location A(Bembridge Marls), with attenuation examined between the seabed and a subbottom reflection $11 \mathrm{~ms}$ beneath (two-way time). (b) Boomer profile over location A (Bembridge Marls), with attenuation examined between the seabed and a subbottom reflection $11 \mathrm{~ms}$ beneath (two-way time). (c) Chirp profile over location B (Headon Beds), with attenuation examined between the seabed and a subbottom reflection $10 \mathrm{~ms}$ beneath (two-way time). (d) Chirp profile over location $\mathrm{C}$ (sands), with attenuation examined between the seabed and a subbottom reflection $7 \mathrm{~ms}$ beneath (two-way time).

\section{Data processing}

We correlate the chirp seismic data with the original source sweep to collapse the waveform correctly (this is not required with boomer data). Further processing to improve interpretability such as filtering, divergence corrections, instantaneous amplitude calculations, and trace mixing is used only to identify the seabed and subbottom reflections and is not applied to the analyzed data. Such processing would modify the spectral content of the data and would be detrimental to the accuracy of the spectral-ratio calculations.

After traces containing suitable reflections are identified, they are windowed within the raw/correlated data and are passed through $1-\mathrm{kHz}$-wide zero-phase Ormsby band-pass filters (with additional $0.5-\mathrm{kHz}$ tapers) that increase in steps of $0.25 \mathrm{kHz}$. The strongest amplitudes from the seabed and subbottom reflections on each trace are extracted from the band-passed time-domain data. The two-way time between the two maximum amplitudes taken from each trace also is calculated, and an amplitude window is passed over a transparent part of the water column to retrieve the background noise floor and assess the signalto-noise content of the measurements.

If no coherent reflections are present close to the subbottom reflector, it might be possible to estimate levels of scattered noise there and use that to assess the signal-to-noise content instead. We found that using frequency bands smaller than $1 \mathrm{kHz}$ generated instability because of the poorer signal-to-noise ratio $(\mathrm{S} / \mathrm{N})$, whereas larger fre- 
quency bands reduce resolution across our frequency range. Zerophase band-pass filters ensure that the energy remains centered on the picked interface, generating the strongest and most precise reflection in the time domain.

\section{Interpretation of spectral-ratio plots}

After the spectral-ratio calculation is applied to traces over a sediment package, two plots are produced. The spectral signature plot (SSP) is a plot of $-\ln \left(A_{R} / A_{S}\right)$ versus $f$ for the mean, median, and standard deviation of all traces within successive $1-\mathrm{kHz}$ frequency bands incrementing by $0.25 \mathrm{kHz}$. The attenuation trend plot (ATP) is a plot of $-\ln \left(A_{R} / A_{S}\right)$ versus $\pi \cdot f \cdot \Delta t_{R}(f)$ for each trace within select frequency bands chosen from the SSP. The ATP shows the best-fitting $Q_{S R}$ model derived using iteratively reweighted robust leastsquares regression.

The SSP is used to identify frequency bands that contain an attenuation trend. A small number of outliers can strongly affect mean data points. However, median data points are robust to such contamination, and it is an indication of good $\mathrm{S} / \mathrm{N}$ when mean and median are similar. Comparing these with the ratio of amplitudes from the water column and seabed reflection $\left(-\ln \left(\right.\right.$ Noise $\left.\left./ A_{S}\right)\right)$ gives an indication of the frequency range at the target reflector that still contains signal, above any background noise. Because of the negative $y$-axis, when the background noise curve is above the mean and median data points, it is weaker than the signal.

We select independent uncontaminated frequency bands for plotting on the ATP using the following empirically derived criteria: The background noise is weaker than two-thirds of the standard deviation range within the frequency band and within the two previous and following frequency bands; the lowest selected uncontaminated frequency band has a central frequency with a power greater than $-30 \mathrm{~dB}$ returned from the seabed; and finally, median frequency band measurements do not follow changes in the background noise curve, confirming that noise is not stronger in the temporal location of the target reflector. Each frequency band on the SSP is $1 \mathrm{kHz}$ wide with central frequencies $0.25 \mathrm{kHz}$ apart. Therefore, the two previous and following frequency bands of the frequency band being considered also are assessed. At least two uncontaminated frequency bands must be selected from the SSP for plotting in the ATP. To be considered independent measurements for statistical purposes, the $1-\mathrm{kHz}$ frequency ranges cannot overlap (ignoring tapers), and to avoid throwing away information, they must define at least the beginning and end of the observed attenuation trend. Any additional uncontaminated frequency bands that lie between, which can be included without producing any overlap in frequency content, should be spaced equally about the center of the attenuation trend.

In the ATP, $Q_{S R}$ is estimated using two statistical fits. First, ordinary least-squares regression is used to fit the data in which all data points are given equal weighting. This is shown on the ATP with its $95 \%$ confidence interval and the intercept value. We also show the correlation coefficient, which is a measure of the accuracy of fit, and the $t$-statistic, which shows the significance of the correlation, by calculating the probability of the correlation coefficient occurring by chance, if the true correlation is zero.

Least-squares estimates are optimal given independent normally distributed errors with constant variance but can behave badly when error distribution is not normal (Fox, 1997). In our case, responses of the data at different frequencies are not of equal quality and do not exhibit constant variance. The variance in errors might increase when frequency is relatively low and little measurable attenuation occurs and/or when frequency is relatively high and the $\mathrm{S} / \mathrm{N}$ is reduced. Therefore, we make a robust estimate of $Q_{S R}$ using an iteratively reweighted robust least-squares regression (see Appendix A). The robust fitting method uses weights to reflect confidence within frequency bands with low variance and to counter error structure within the data during the fitting process. Thus, the robust $Q_{S R}$ remains stable in the presence of high-leverage outliers near the extremities of the fitted curve and should be the most accurate and unbiased result.

\section{EXPERIMENTAL RESULTS}

\section{Solent location A}

From location A, we estimate the $Q_{S R}$ of a sediment package that extends $11 \mathrm{~ms}$ (two-way time) beneath the seabed (Figure 3a and b). SSPs from the two chirp surveys (Figure $4 a$ and c) show the same trends and the same uncontaminated frequency range between $2.00 \mathrm{kHz}$ and $5.00 \mathrm{kHz}$. Below $2.00 \mathrm{kHz}$, the mean and median residuals follow the shape of the background noise curve. Above $5.00 \mathrm{kHz}$, the background noise becomes stronger than the signal
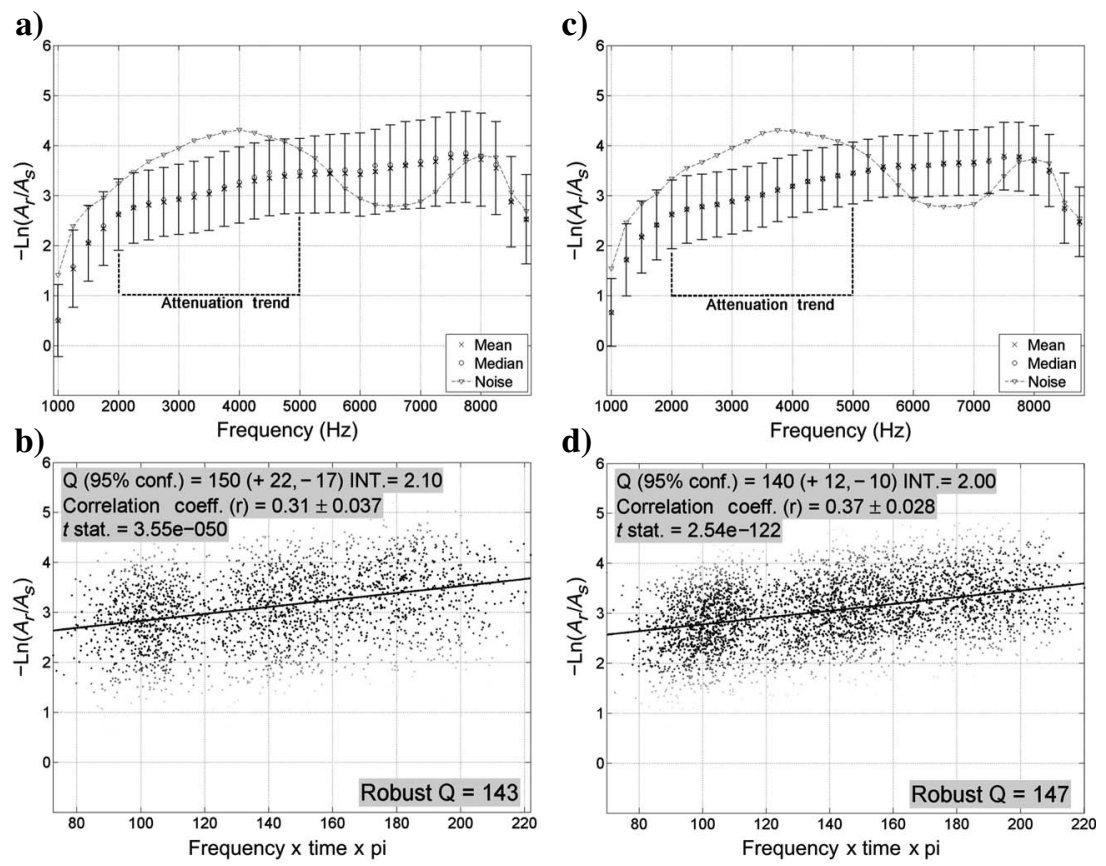

Figure 4. Results from location A (Bembridge Marls): (a) Chirp southwest-northeast SSP (750 traces) showing attenuation trend $2.00-5.00 \mathrm{kHz}$. (b) Chirp southwest-northeast ATP using frequency bands $2.50,3.50$, and $4.50 \mathrm{kHz}$. (c) Chirp northeast-southwest SSP (1250 traces) showing attenuation trend 2.00-5.00 kHz. (d) Chirp northeast-southwest ATP using frequency bands 2.50, 3.50, and $4.50 \mathrm{kHz}$. Data points on ATPs are colored black-white according to their weighting for the robust regression $(1-0)$. 
a)

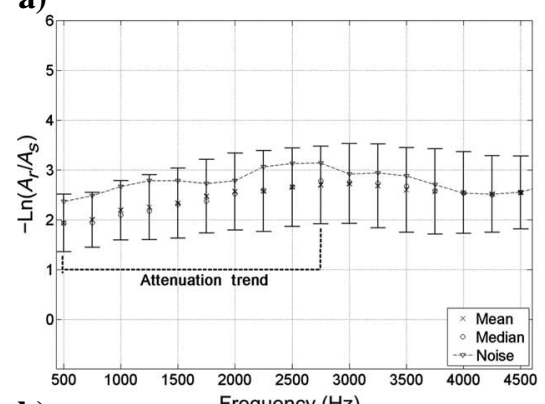

b)

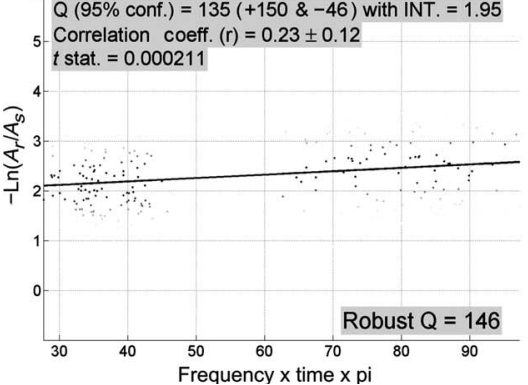

c)

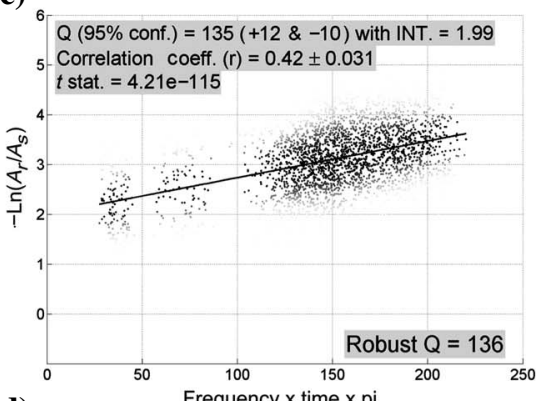

d)

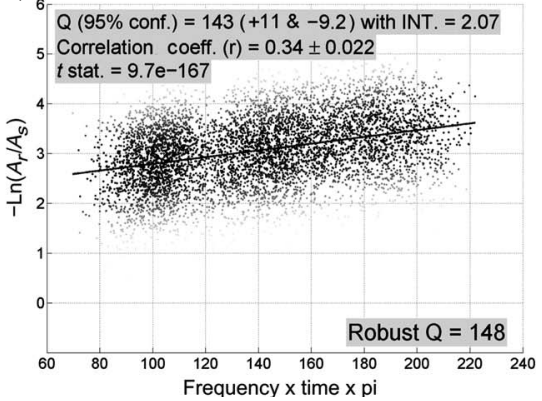

Figure 5. Results from location A (Bembridge Marls): (a) Boomer SSP showing attenuation trend $0.50-2.75 \mathrm{kHz}$. (b) Boomer ATP using 1.00 - and $2.25-\mathrm{kHz}$ frequency bands. (c) combined chirp northeast-southwest (1250 traces) and boomer ATP using frequency bands $1.00,2.00,3.50$, and $4.50 \mathrm{kHz}$. (d) Combined chirp northeast-southwest (1250 traces) and chirp southwest-northeast (750 traces) ATP.

a)

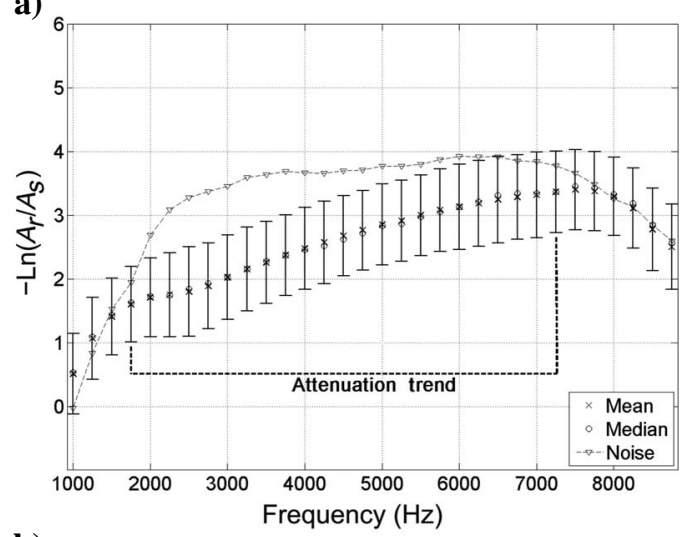

b)

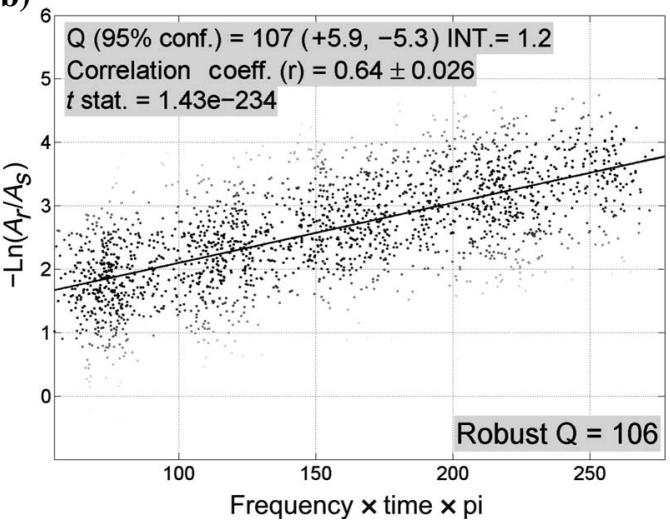

Figure 6. Results from location B (Headon Beds): (a) Chirp SSP exhibiting an uncontaminated attenuation trend $1.75-7.25 \mathrm{kHz}$ and $(\mathrm{b})$ ATP using frequency bands $2.25,3.25,4.50,5.75$, and $6.75 \mathrm{kHz}$ and showing the robust $Q_{S R}$ for these sediments. from the subbottom reflector. From these attenuation trends, the $1-\mathrm{kHz}$ uncontaminated frequency bands centered on $2.50,3.50$, and $4.50 \mathrm{kHz}$ are selected for use in the ATPs. Thus, over a frequency range of $2.00-5.00 \mathrm{kHz}$, a linear regression line fits the data within the ATPs (Figure $4 \mathrm{~b}$ and d) with frequency-independent robust $Q_{S R} \mathrm{~s}$ of 143 and 147 . Both robust $Q_{S R}$ S fall within the predicted $95 \%$ confidence intervals of $150(+22 ;-17)$ and $140(+12 ;-10)$, respectively. However, the chirp northeast-southwest survey has better precision and a higher correlation of 0.37 because of the greater number of traces over the same distance resulting from the tidal flow direction.

Figure $5 \mathrm{a}$ and $\mathrm{b}$ shows results from applying this spectral-ratio technique to the boomer data over the same sediment package. The SSP in Figure 5a shows that the $\mathrm{S} / \mathrm{N}$ within the boomer data is inferior to chirp data, but an uncontaminated frequency range is observed between 0.50 $\mathrm{kHz}$ and $2.75 \mathrm{kHz}$. Above $2.75 \mathrm{kHz}$, noise contaminates the ratios, and at $4.00 \mathrm{kHz}$, the boomer power spectrum falls below $-30 \mathrm{~dB}$, allowing the background noise to become stronger than the signal, and the two converge. Therefore, from this attenuation trend, the $1-\mathrm{kHz}$ uncontaminated frequency bands centered on $1.00 \mathrm{kHz}$ and 2.25 $\mathrm{kHz}$ are selected for use in the ATP. Over the frequency range of $0.50-2.75 \mathrm{kHz}$, we use a linear-regression line to fit the data within the ATP (Figure 5b) and find a frequency-independent robust $Q_{S R}$ of 146 . The robust $Q_{S R}$ falls within the $95 \%$ confidence interval of $135(+150 ;-46)$ and has a correlation of 0.23 . This is consistent with the chirp results, but the $95 \%$ confidence interval is poor because there are fewer traces over the sediment package resulting from the slower firing rate of the boomer source and because of a reduced frequency range relative to the chirp data.

Combining the $1.0-$ and $2.0-\mathrm{kHz}$ frequency bands from the boomer and the 3.5- and $4.5-\mathrm{kHz}$ frequency bands from the northeast-southwest chirp enables $Q_{S R}$ to be examined from 0.5 to $5.0 \mathrm{kHz}$ and produces the ATP in Figure 5c. The addition of the boomer data derives a superior result, with a frequency-independent robust $Q_{S R}$ of 136 that lies within a precise $95 \%$ confidence interval of $135(+12 ;-10)$ and has a correlation coefficient of 0.42. Combining the southwest-northeast and northeast-southwest chirp data sets (Figure 5d) gives a 95\% confidence interval of $(+11 ;-9)$, which is an improvement over that achieved by the northeast-southwest chirp data alone. However, the correlation coefficient of 0.34 lies between the correlations achieved by the two data sets individually. Therefore, the more accurate result is from the combination of the boomer data with the northeastsouthwest survey, which provided the extended frequency range. The zero values of the $t$-statistic show that all the correlations are statistically significant and that a linear fit is appropriate over this frequency range.

\section{Solent location B}

From location B, we estimate the $Q_{S R}$ of a sediment package that extends $10 \mathrm{~ms}$ (two-way time) beneath the seabed (Figure 3c). The SSP in Figure 6a shows an uncontaminated frequency range be- 
tween $1.75 \mathrm{kHz}$ and $7.25 \mathrm{kHz}$. Below $1.75 \mathrm{kHz}$ and above 7.25 $\mathrm{kHz}$, the background noise becomes stronger than the signal from the subbottom reflector. From this attenuation trend, $1-\mathrm{kHz}$ uncontaminated frequency bands centered on 2.25, 3.25, 4.50, 5.75, and $6.75 \mathrm{kHz}$ are selected for use in the ATP. Over the frequency range of $1.75-7.25 \mathrm{kHz}$, we find that a linear-regression line fits the data within the ATP (Figure 6b) with a frequency-independent robust $Q_{S R}$ of 106 . The robust $Q_{S R}$ falls within the precise $95 \%$ confidence interval of $107(+6 ;-5)$. The $t$-statistic is zero, confirming the linear fit and implying that the correlation coefficient of 0.64 is significant.

\section{Solent location C}

From location C, we estimate the $Q_{S R}$ of a sediment package that extends $7 \mathrm{~ms}$ (two-way time) below the seabed (Figure 3d). The SSP in Figure 7a shows an uncontaminated frequency range between $1.75 \mathrm{kHz}$ and $7.25 \mathrm{kHz}$. Below $1.75 \mathrm{kHz}$, the effective chirp power spectrum falls toward $-50 \mathrm{~dB}$, and we observe increased standard deviation about the mean ratio. Above $7.25 \mathrm{kHz}$, the background noise becomes stronger than the signal from the subbottom reflector. From this attenuation trend, 1-kHz uncontaminated frequency bands centered on $2.25,3.25,4.50,5.75$, and $6.75 \mathrm{kHz}$ are selected for use in the ATP. Over the frequency range of $1.75-7.25 \mathrm{kHz}$, we find that a linear-regression line fits the data within the ATP (Figure 7b) with a frequency-independent robust $Q_{S R}$ of 66 . The robust $Q_{S R}$ falls within the $95 \%$ confidence interval of $63(+10 ;-7)$. The $t$-statistic is

a)

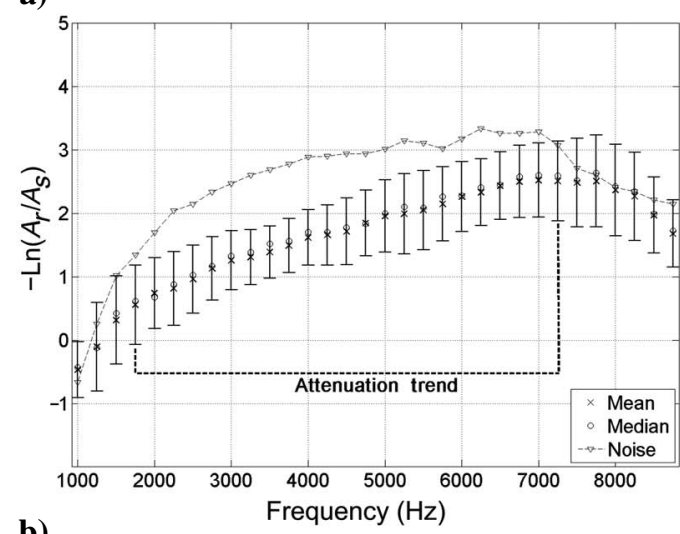

b)

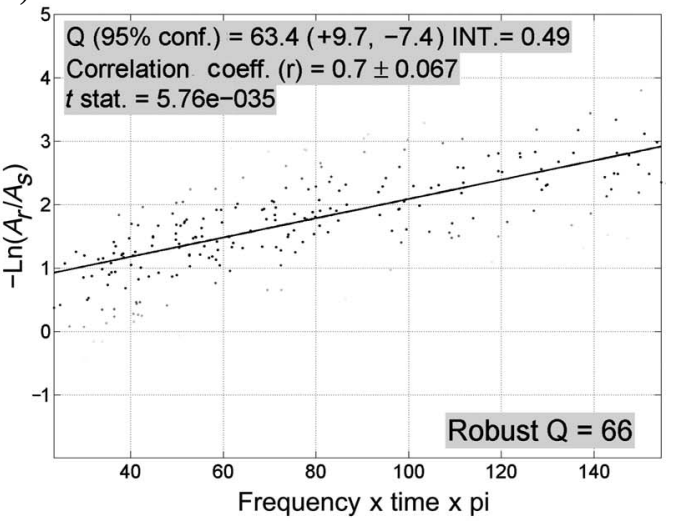

Figure 7. Results from location C (sands): (a) Chirp SSP exhibiting an uncontaminated attenuation trend $1.75-7.25 \mathrm{kHz}$ and (b) ATP using frequency bands $2.25,3.25,4.50,5.75$, and $6.75 \mathrm{kHz}$ and showing the robust $Q_{S R}$ for these sediments. zero, confirming the linear fit and implying that the correlation coefficient of 0.7 is significant.

\section{DISCUSSION}

Robust estimates of $Q_{S R}$ all converge within the $95 \%$ confidence intervals about the ordinary least-squares $Q_{S R}$, implying that a Gaussian error structure is reasonable and that the $95 \%$ confidence limits produced are meaningful. The $t$-statistics indicate that all $Q_{S R}$ fits are appropriate and correlations, which range from 0.23 to 0.70 , are significant. SSPs makes full use of the power-spectrum information, so any variation of $Q_{S R}$ with frequency above background noise levels is identified and evaluated robustly. We showed that it is simple to combine the high-resolution seismic data from alternative sources, allowing us to observe $Q_{S R}$ variation over a broader frequency range. The successful combination of boomer and chirp data also confirms that all the source/receiver responses (which would be different between the chirp and boomer surveys) are eliminated from measurements when ratios are taken. Figure 8 reviews the application of our spectral-ratio technique.

We have tested how the number of observations (traces multiplied by independent frequency bands) affects the ordinary least-squares regression estimate of $Q_{S R}$ and the corresponding $95 \%$ confidence interval, using the chirp northeast-southwest survey data from location A. By keeping the same three independent frequency bands used previously (measuring attenuation between $2.0 \mathrm{kHz}$ and $5.0 \mathrm{kHz}$ ) and selecting successively longer sections about a common center point, we show that precision and correlation increase with the number of traces (Figure 9). Adding the $Q_{S R}$ derived from the combination of chirp northeast-southwest data with boomer survey data (measuring attenuation between $0.5 \mathrm{kHz}$ and $5.0 \mathrm{kHz}$ ), we see that fewer observations are required to achieve an equivalent $95 \%$ confidence interval of $+12 ;-10$, and a more accurate fit with a correlation coefficient of 0.42 is produced.

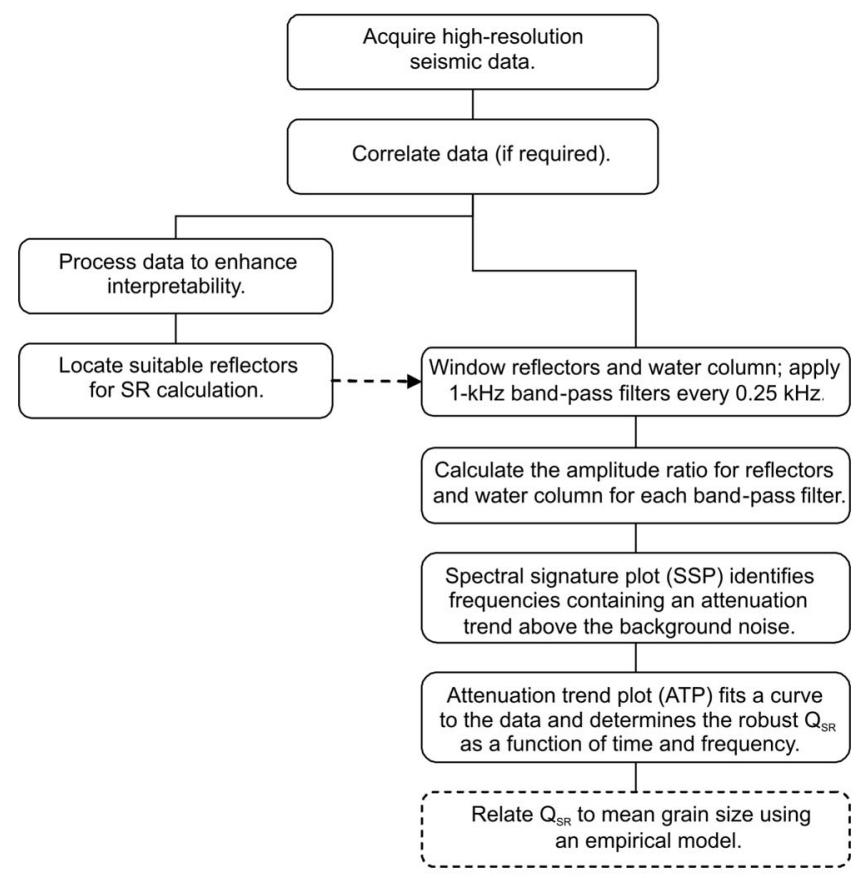

Figure 8. Flow diagram for the spectral-ratio technique presented in this paper. 
The result from combining the southwest-northeast and northeast-southwest chirp survey data looks slightly anomalous because of the reduction in correlation; this is because it is unlikely that the lines are exactly coincident. Therefore, the accuracy in the $Q_{S R}$ fit is dependent on bandwidth and slightly dependent on precision (Figure 9). Precision is dependent on the variation in observed attenuation, which depends on $\Delta t_{R}(f)$ and $Q(f)$.

It follows that with sediments in which attenuation is weaker and more observations are used, such as the Bembridge Marls (7.7 phi) in location A, greater benefit is obtained from using iteratively reweighted robust least-squares regression to subdue the effects of outliers. The two independent chirp surveys and the boomer survey found the robust $Q_{S R}$ to be 143,147, and 146, respectively, although the respective $Q_{S R}$ and $95 \%$ confidence intervals from the ordinary least-squares regression were $150(+22 ;-17), 140(+12 ;-10)$, and $135(+150 ;-46)$.

Comparison of chirp SSPs from the three locations shows that the standard deviation in each frequency band reduces as $Q_{S R}$ reduces. Therefore, more traces were required over the sediment packages at locations $\mathrm{A}$ and $\mathrm{B}$ relative to $\mathrm{C}$ to achieve a desirable $95 \%$ confidence interval. Location A shows the greatest standard deviation in the frequency bands, possibly because of the poorer sorting of the sediment and the seawater-gravel interface. Precision diminishes as $Q_{S R}$ increases because $1 / Q(f)$ (the gradient of the fitted curve) becomes smaller and the fit becomes more ambiguous. White (1992) confirms that $Q$ is estimated most easily when the effect of attenuation is large. Consequently, to achieve a desirable $95 \%$ confidence interval of about \pm 10 for the $Q_{S R}$ of the Bembridge Marls (7.7 phi), at least 1250 traces using only chirp data are required. This equates to the complete 400-m section examined over the Bembridge Marls, much greater than the 200- and 25-m sections necessary over the bettersorted lower $Q_{S R}$ sediments at locations B and C. Thus, the length scales over which lateral homogeneity is required are relatively short.

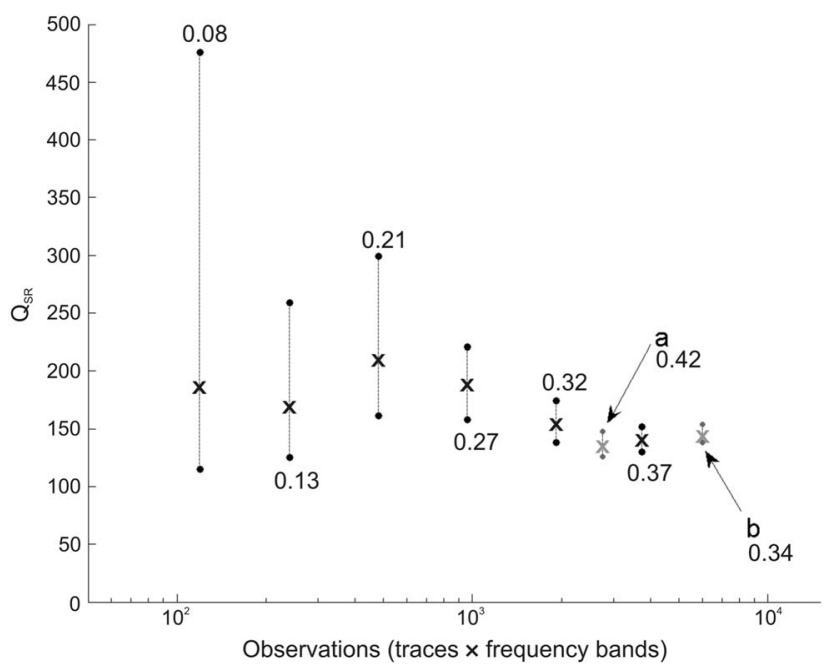

Figure 9. Graph showing 95\% confidence (black dots) and predicted $Q_{S R}$ (crosses) calculated using linear ordinary least-squares regression against the number of observations over the sediment at location A about a common midpoint, from the northeast-southwest chirp data: (a) locates the result from the addition of the boomer data, and (b) locates the result from the addition of the southwest-northeast chirp data.
Examining how noise affects the frequency ranges at the three locations, we see that above $8.00 \mathrm{kHz}$ within all chirp SSPs, the signal from the subbottom reflector has become attenuated completely and begins to follow the background noise curve. This noise contamination at higher frequencies causes attenuation trends to effectively flatten out, which also is observed in synthetic examples by Matheney and Nowack (1995). Chirp SSPs from location A (Figure 4a and c) show that the ratios with the subbottom reflector could be contaminated with an increase in background noise at $5 \mathrm{kHz}$. Although the background noise measured from the water column is stronger at 5-8 kHz, the amplitudes from the subbottom reflector are being taken from a different temporal location where this noise has a reduced effect. The lower $Q_{S R}$ of the sediment packages from locations B and $\mathrm{C}$ allow a measurable amount of attenuation to take place at 1.75 $\mathrm{kHz}$, as opposed to location A, where the higher $Q_{S R}$ causes less significant attenuation below $2 \mathrm{kHz}$ and the measurements become sensitive to background noise.

The constant $Q_{S R}$ fits for the three substrates suggest that intrinsic attenuation, similar to that explained by the G-S theory (Buckingham, 2000) for unconsolidated sediments, is dominant over scattering attenuation within our frequency range. Near-linear scaling of compressional-wave attenuation with frequency has been identified by other researchers. Hamilton (1972) combined his results with others and observed compressional-wave attenuation to scale with frequency to a power close to unity between $5 \mathrm{kHz}$ and $1000 \mathrm{kHz}$ within unconsolidated sands, clays, and mixed sediments. Bowles (1997) compiled experiments in unconsolidated fine-grained sediments spanning $10 \mathrm{~Hz}$ to $500 \mathrm{kHz}$ (although without observations in the interval $0.6-4.0 \mathrm{kHz}$ ) and found a similar scaling with frequency to the power of 1.12. Williams et al. (2002) report a linear scaling of compressional-wave attenuation with frequency in sandy sediment from the SAX99 site from $1.7 \mathrm{kHz}$ to $400 \mathrm{kHz}$. Our results in finegrained sediment between $0.50 \mathrm{kHz}$ and $5.00 \mathrm{kHz}$ support Bowles' (1997) conclusion.

Our observations in sandy sediments between $1.75 \mathrm{kHz}$ and $7.25 \mathrm{kHz}$ suggest that a near-linear scaling of attenuation with frequency can be extended to coarse grain-dominated sediments. Furthermore, this argues for the validity of using empirical models generated from higher-frequency laboratory attenuation studies to relate lower-frequency remote attenuation measurements to sediment physical properties.

Figure 10 compares our observations with a compilation of attenuation studies over a wide range of sediments from the San Diego, California, area (Shumway, 1960; Hamilton, 1972). The most accurate robust $Q_{S R}$ of 136 from location A for the Bembridge Marls (7.7 phi) is consistent with the higher $Q$ observations in the model. The relatively thin layer of gravel above the Bembridge Marls had little effect on the overall attenuation. The robust $Q_{S R}$ of 106 for the Headon Beds (6.4 phi) from location B conforms with the curve defined by the San Diego observations, and the lowest robust $Q_{S R}$ of 66 from the Bramble Bank sands (2.5 phi) from location $\mathrm{C}$ is generally consistent with lower $Q$ observations in the model. It is apparent that $Q$ is not diagnostic below 6 phi and a $Q$ of 75 , although other properties such as compressional-wave velocity might help to resolve these coarse grain-dominated sediments (e.g., Hamilton, 1972). Our results and the majority of observations from San Diego suggest that $Q$ becomes greater than 75 after 6 phi, the point where larger grains become suspended in a clay matrix (McCann and McCann, 1969). 


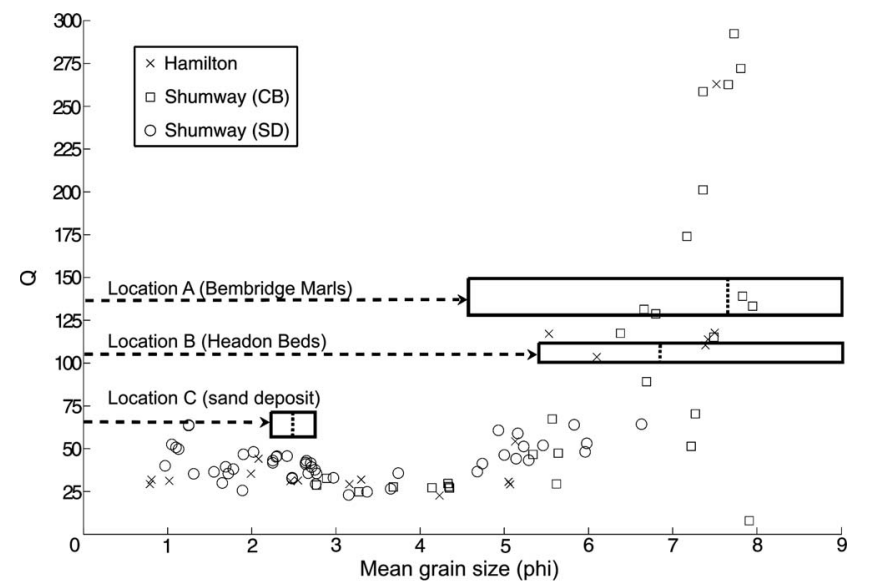

Figure 10. $Q$ versus mean grain size (phi) of sediments from the San Diego area (Shumway, 1960; Hamilton, 1972, his Tables 1 and 2). Dashed lines locate the Solent robust $Q_{S R}$ fits. Solid boxes represent the $95 \%$ confidence interval in the ordinary least-squares regression and the sorting of sediment. Vertical dashed lines represent the mean grain size of the sediments.

\section{CONCLUSION}

The spectral-ratio technique presented here for seismic-reflection data shows that a frequency-independent $Q_{S R}$ is the most appropriate fit to unconsolidated sand- and clay-dominated sediments in the Solent over the frequency range $0.5-8.0 \mathrm{kHz}$. The linearity of compressional-wave attenuation with frequency leads us to conclude that intrinsic attenuation is dominant over scattering attenuation within this frequency range, for these sediment packages.

For sediment packages that have prerequisite conditions of lateral homogeneity and isolated subhorizontal reflections above the first seabed multiple, high-resolution seismic sources can estimate the $Q_{S R}$ with a $95 \%$ confidence interval of about \pm 10 , with a significant correlation coefficient. Estimating $Q_{S R}$ in clay-dominated sediment packages, where attenuation is weaker and the $Q_{S R}$ fit is more ambiguous, requires more observations to improve the $95 \%$ confidence interval and benefits from the use of iteratively reweighted robust least-squares regression to subdue outliers. If possible, data acquired using two seismic sources, such as a chirp and boomer sonar, can be combined to extend the frequency range over which attenuation is analyzed and to improve the confidence and correlation of the $Q_{S R}$ fit.

$Q$ values provide a limited ability to differentiate mean grain sizes. For a $Q$ less than 75 , the sediment is likely to be coarse graindominated with a mean grain size ranging from 1 phi to 6 phi. For a $Q$ over 75 , the sediment is likely to be clay dominated with a mean grain size greater than 6 phi.

\section{ACKNOWLEDGMENTS}

The authors acknowledge funding from the Natural Environment Research Council (NERC) and the Joint Research Equipment Initiative for completion of this work. Luke Pinson was supported by an NERC studentship (13978). We are grateful for the enthusiasm and expertise at sea of J. Davis, G. Etheridge, and R. Wilkie. We also thank three anonymous reviewers and Michael Asten, associate editor, for their constructive comments that have improved the manuscript.

\section{APPENDIX A}

\section{ROBUST REGRESSION}

Statistical packages often feature robust regression as part of their curve-fitting toolboxes. The following is the implementation used by MATLAB ${ }^{\circledR}$ (MathWorks, Inc., 2007).

The initial sum of the squared residuals $S$ has the residuals (fitted response values $\hat{y}$ deducted from the observed response values $y_{i}$ ) weighted $w_{f}$ according to the variance within their frequency bands $\sigma_{f}^{2}$.

$$
\begin{gathered}
S=\sum_{i=1}^{n} w_{f} \\
w_{f}=\frac{1}{\sigma_{f}^{2}} .
\end{gathered}
$$

Adjusted residuals $r_{\text {adj }}$ are computed according to their leverages $h_{i}$, in which high-leverage data are downweighted to reduce their effect on the least-squares fit,

$$
r_{\text {adj }}=\frac{y_{i}-\hat{y}_{i}}{\sqrt{1-h_{i}}} .
$$

The adjusted residuals are standardized $(u)$ using the product of a tuning constant $K(4.685)$ and the robust variance $s$, which is the median absolute residual (a robust measure of spread) divided by 0.6745 . The values stated are picked to give reasonably high efficiency in the case of a normal error distribution and still offer protection against outliers when using a bisquare weight function (Fox, 1997),

$$
u=\frac{r_{\mathrm{adj}}}{K \cdot s} .
$$

Bisquare weights then are calculated as a function of $u$. The weights decline as soon as the error departs from 0 and become 0 when the absolute error becomes larger than $K \cdot s$. The new weights $\left(W_{i}\right)$ for the first robust regression are the product of $w_{f}$ and $w_{i}$,

$$
\begin{aligned}
& w_{i}=\left\{\begin{array}{cc}
\left(1-\left(u_{i}\right)^{2}\right)^{2} & \left|u_{i}\right|<1 \\
0 & \left|u_{i}\right| \geq 1
\end{array}\right. \\
& W_{i}=w_{f} \cdot w_{i} \cdot w_{i+n} \ldots
\end{aligned}
$$

After each iteration, $W_{i}$ is the product of the previous weights and the recalculated $w_{i}$ from repeating equations A-3 to A-5. The process continues until the residuals converge.

\section{REFERENCES}

Båth, M., 1974, Spectral analysis in geophysics: Developments in solid earth geophysics: Elsevier Science Ltd.

Biot, M. A., 1956a, Theory of propagation of elastic waves in a fluid-saturated porous solid. I: Low-frequency range: Journal of the Acoustical Society of America, 28, 168-178.

-1956b, Theory of propagation of elastic waves in a fluid-saturated porous solid. II: Higher frequency range: Journal of the Acoustical Society of America, 28, 179-191.

Bowles, F. A., 1997, Observations on attenuation and shear-wave velocity in fine-grained, marine sediments: Journal of the Acoustical Society of 
America, 101, 3385-3396.

Buchanan, J., 2006, A comparison of broadband models for sand sediments Journal of the Acoustical Society of America, 120, 3584-3598.

Buckingham, M. J., 2000, Wave propagation, stress relaxation, and grain-tograin shearing in saturated, unconsolidated marine sediments: Journal of the Acoustical Society of America, 108, 2796-2815.

Bull, J. M., R. Quinn, and J. K. Dix, 1998, Reflection coefficient calculation from marine high resolution seismic reflection (chirp) data and application to an archaeological case study: Marine Geophysical Researches, 20, $1-11$

Dvorkin, J., and A. Nur, 1993, Dynamic poroelasticity: A unified model with the squirt and the Biot mechanisms: Geophysics, 58, 524-533.

Fox, J., 1997, Applied regression analysis, linear models, and related methods: SAGE Publications.

Guigné, J. Y., N. G. Pace, and V. H. Chin, 1989, Dynamic extraction of sediment attenuation from subbottom acoustic data: Journal of Geophysical Research, 94, 5745-5755.

Gutowski, M., J. Bull, T. Henstock, J. Dix, P. Hogarth, T. Leighton, and P. White, 2002, Chirp sub-bottom profiler source signature design and field testing: Marine Geophysical Researches, 23, 481-492.

Hamilton, E., 1972, Compressional wave attenuation in marine sediments: Geophysics, 37, 620-646.

Hosken, J. W. J., S. K. Mayo, and A. T. Walden, 1992, The influence of reflection coefficient statistics on the seismic method: Scattering attenuation and transmission wavelets: Proceedings of the Royal Society of London A, 439, $1-23$.

Jannsen, D., J. Voss, and F. Theilen, 1985, Comparison to determine $Q$ in shallow marine sediments from vertical reflection seismograms: Geophysical Prospecting, 33, 479-497.

LeBlanc, L. R., S. Panda, and S. G. Schock, 1992, Sonar attenuation modelling for classification of marine sediments: Journal of the Acoustical Society of America, 91, 116-126.

Matheney, M. P., and R. L. Nowack, 1995, Seismic attenuation values obtained from instantaneous-frequency matching and spectral ratios: Geophysics Journal International, 123, 1-15.

MathWorks, Inc., 2007, Robust regression: Curve fitting toolbox, www.mathworks.com/access/helpdesk_r13/help/toolbox/curvefit/ ch_fitt5.htm, accessed January 26, 2008.

McCann, C., and D. McCann, 1969, The attenuation of compressional waves in marine sediments: Geophysics, 34, 882-891.

Muller, T. M., and S. A. Shapiro, 2004, Scattering attenuation in randomly layered structures with finite lateral extent: A hybrid $Q$ model: Geophysics, 69, 1530-1534.

O’Doherty, R. F., and N. A. Anstey, 1971, Reflections on amplitudes: Geophysical Prospecting, 19, 430-458.

Schock, S. G., 2004a, A method for estimating the physical and acoustic properties of the sea bed using chirp sonar data: IEEE Journal of Oceanic
Engineering, 29, 1200-1217.

,$- 2004 \mathrm{~b}$, Remote estimates of physical and acoustic sediment properties in the South China Sea using chirp sonar data and the Biot model IEEE Journal of Oceanic Engineering, 29, 1218-1230.

Schock, S. G., and L. R. LeBlanc, 1990, Chirp sonar: New technology for sub-bottom profiling: Sea Technology, 31, 35-43.

Shapiro, S. A., and P. Hubral, 1999, Elastic waves in random media: Springer Publishing Company, Inc.

Shumway, G., 1960, Sound speed and absorption studies of marine sediments by a resonance method: Geophysics, 25, 451-467.

Simpkin, P. G., and A. Davis, 1993, For seismic profiling in very shallow water: A novel receiver: Sea Technology, 34, 21-28.

Simpson, H. J., and B. H. Houston, 2000, Synthetic array measurements of acoustical waves propagating into a water-saturated sandy bottom for a smoothed and a roughened interface: Journal of the Acoustical Society of America, 107, 2329-2337.

Simpson, H. J., B. H. Houston, S. W. Liskey, P. A. Frank, A. R. Bedroz, L. R. Kraus, C. K. Frederickson, and S. Stanic, 2003, At-sea measurements of sound penetration into sediments using a buried vertical synthetic array: Journal of the Acoustical Society of America, 114, 1281-1290.

Stevenson, I. R., C. McCann, and P. Runciman, 2002, An attenuation-based sediment classification technique using chirp sub-bottom profiler data and laboratory acoustic analysis: Marine Geophysical Researches, 23, 277-298.

Stoll, R. D., 1974, Acoustic waves in saturated sediments, in L. Hampton, ed., Physics of sound in marine sediments: Plenum Press, 19-39.

2002, Velocity dispersion in water-saturated granular sediment: Journal of the Acoustical Society of America, 111, 785-793.

Thorsos, E., K. Williams, N. Chotiros, J. Christoff, K. Commander, C. Greenlaw, D. Holliday, D. Jackson, J. Lopes, D. Maghee, J. Piper, M. Richardson, and D. Tang, 2001, An overview of SAX99: Acoustic measurements: IEEE Journal of Oceanic Engineering, 26, 4-25.

Turgut, A., and T. Yamamoto, 1990, Measurements of acoustic wave velocities and attenuation in marine sediments: Journal of the Acoustical Society of America, 87, 2376-2383.

Velegrakis, A., 2000, Geology, geomorphology and sediments of the Solent system, in M. B. Collins and K. Ansell, eds., Solent science - A review: Proceedings in Marine Science, 1, 21-43.

West, I. M., 1980, Geology of the Solent estuarine system: NERC Publication Series C, 22, 6-12.

White, R. E., 1992, The accuracy of estimating $Q$ from seismic data: Geophysics, 57, 1508-1512.

Williams, K., D. Jackson, E. Thorsos, D. Tang, and S. Schock, 2002, Comparison of sound speed and attenuation measured in a sandy sediment to predictions based on the Biot theory of porous media: IEEE Journal of Oceanic Engineering, 27, 413-428. 